\title{
The Effects of Nutrition, Exercise, and a Praying Program on Reducing Iron Overload in Patients With Beta-Thalassemia Major: A Randomized Clinical Trial
}

\author{
Zahra Molazem, ${ }^{1,}$ Roghaye Noormohammadi, ${ }^{2}$ Roya Dokouhaki, ${ }^{3}$ Maryam Zakerinia, ${ }^{4}$ and Zahra
}

Bagheri ${ }^{5}$

${ }^{1}$ Community Based Nursing and Midwifery Research Center, Faculty of Nursing and Midwifery, Shiraz University of Medical Sciences, Shiraz, IR Iran

${ }^{2}$ Student Research Committee, Shiraz University of Medical Sciences, Shiraz, IR Iran

${ }^{3}$ Department of Nursing, Shiraz University of Medical Sciences, Shiraz, IR Iran

${ }^{4}$ Department of Internal Medicine, Hematology Research Center, Shiraz University of Medical Sciences, Shiraz, IR Iran

${ }^{5}$ Department of Biostatistics, Shiraz University of Medical Sciences, Shiraz, IR Iran

"Corresponding author: Zahra Molazem, Community Based Nursing and Midwifery Research Center, Faculty of Nursing and Midwifery, Shiraz University of Medical Sciences, Shiraz, IR Iran. Tel: +98-9177154036, Fax: +98-7116474252, E-mail: molazem@sums.ac.ir

Received 2015 August 17; Revised 2016 April 20; Accepted 2016 May 08.

\begin{abstract}
Background: Excessive iron accumulation in the visceral organs creates problems for patients with beta-thalassemia major. Despite chelation therapy, mortality rate from the complications of this disease is still quite high.

Objectives: This study aimed to investigate the effectiveness of nutrition, exercise, and a praying program at reducing iron overload in patients with beta-thalassemia major.

Patients and Methods: This randomized clinical trial assessed the effect of the designed care program on iron overload. The study was conducted in 38 patients with beta-thalassemia major who ranged in age from 15 - 35 years and had been referred to the largest center for thalassemic patients in Shiraz. The patients were randomly assigned to an intervention $(\mathrm{n}=18)$ and a control $(\mathrm{n}=20)$ group. Blood samples were collected from the participants before and two months after the intervention. Then, the data were statistically analyzed using chi-square, Fisher's exact test, Mann-Whitney U-test, Wilcoxon, independent samples t-test, and paired samples t-test.

Results: The results showed that the mean level of serum ferritin significantly decreased in the intervention group two months after beginning the intervention. Also, the mean level of serum iron decreased in the intervention group, but the difference was not statistically significant.

Conclusions: The planned educational program could be used to reduce iron overload and ultimately improve the patients' health status.
\end{abstract}

Keywords: Beta-Thalassemia Major, Exercise, Iron, Ferritin, Nutrition, Praying

\section{Background}

Thalassemia is a genetic disease in which oxygen is not properly supplied to bodily organs due to an abnormal structure of the patient's hemoglobin (1). The disease is seen in almost all human races, but it is more prevalent in the Mediterranean and tropical regions as well as in Asia and Africa $(1,2)$. The annual incidence rate of symptomatic cases has been estimated to be 1 in 100,000 populations worldwide and 1 in 10,000 in European countries (3). Iran, with approximately 3 million minor thalassemic and 25 thousand major thalassemic patients, is located in the thalassemia belt (2). Repeated blood transfusions and iron absorption from the intestine may cause iron overload in these patients (4). Iron overload can, in turn, lead to increased levels of free radicals in the body. Studies have indicated that the level of oxidative stress is significantly high in patients with transfusion-dependent thalassemia (5-7)], while the levels of antioxidants, such as vitamins $\mathrm{E}$ and $\mathrm{C}$, are lower than the normal range $(8,9)$.

Excess iron is precipitated in the visceral organs, primarily the heart, liver, and endocrine glands (1). Despite the availability of iron-chelators, heart toxicity remains the leading cause of death in beta-thalassemia major patients due to iron overload (10-12). The most common treatment method for the removal of excessive iron is chelation therapy (1). Desferal is a medicine in this category; however, it 
has several side effects, such as excessive pain during injection (13). The quality of its oral form is also challenging, and it is not applicable to all patients (14). Moreover, with the rising cost of desferal, a high economic burden is imposed on society's healthcare system. Thus, attempts to identify appropriate and cost-effective ways to reduce iron overload in patients with beta-thalassemia were among the researchers' top concerns. Nevertheless, few studies have been conducted in this area so far. In an investigation by Schumacher et al., iron depletion was reported in 10\% of male and $20 \%$ of female athletes (15). Furthermore, some researchers assessed the effects of aerobic exercise and intercessory prayer on ferritin in patients with thalassemia $(2,16)$. Other researchers, by determining the nutritional needs of these patients and suggesting the addition of specific complements to the treatment program, have tried to meet the nutritional needs of these patients and reduce tissue damage due to iron overload (17). Yet, only a limited number of studies have been conducted in this regard, particularly in Iran.

\section{Objectives}

Therefore, the present study aimed to investigate the effect of nutrition, exercise, and a prayer program on reducing iron overload in patients with beta-thalassemia major.

\section{Patients and Methods}

\subsection{Study Design}

This randomized clinical trial with pre/posttest design was performed on two groups of thalassemia patients (intervention and control groups).

\subsection{Setting and Participants}

This study was conducted in Shahid Dastgheib hospital affiliated with Shiraz University of Medical Sciences, the largest center for thalassemia patients in Shiraz. The study was performed in thalassemia patients from May to October 2013. The inclusion criteria for the study were being between 15 and 35 years of age, having the ability to receive the training and take care of oneself, not being prohibited to exercise by a cardiologist, and not having participated in similar studies during the past six months. On the other hand, the exclusion criteria were a lack of cooperation during the study (unwillingness, migration, or non-compliance with the program), hospitalization for any reason (acute complications of the disease, cardiovascular disorders, and other problems), suffering from acute or chronic infectious diseases, and an urgent need to alter the chelation therapy due to an excessive increase or decrease in iron overload.

Based on similar studies (16), a 30-subjects sample size was determined to be adequate for the study (15 patients in each group). However, considering the probability of loss, the sample size was increased to 50 subjects ( 25 in each group). Among the 390 patients with thalassemia who had been referred to the hospital, 200 did not meet the inclusion criteria and 100 were not willing to participate in the study. Therefore, 50 patients with major thalassemia were selected based on the inclusion criteria of the study (Figure 1). These patients were randomly divided into an intervention and a control group. In this study, 12 patients were excluded due to hospitalization, unwillingness to continue participating in the study, and suffering from infectious diseases. Thus, the number of patients in the intervention and control groups decreased from 25 each to 18 and 20, respectively.

\subsection{Interventions}

The intervention group participants were divided into groups of five for training classes. The educational content was prepared based on various resources, articles, and specialists' opinions. In the first session, the group members became familiar with each other, expressed their goals, and talked about their life experiences. The patients' educational needs were also determined using a questionnaire.

In the second session, the patients were provided with a brief description of the types of the disease, new treatment methods, and the dos and don'ts of eating. Considering nutrition, the focus was on avoiding heme-ironrich foods (iron found in meat), substitution of the nutritional needs with non-heme-iron-rich foods (iron found in non-meat materials), and the consumption of antioxidants, such as vitamins E and C (Box 1).

In the third session, exercise and physical activity within one's physical endurance were emphasized, and the principles and methods were explained. In addition, the patients were asked to do moderate exercises, such as walking, at home for 15 minutes every day and increase or decrease their duration and speed depending on their tolerance (Box 2).

In the fourth session, the importance of preventing infection, the association between infection, iron, and iron chelators as well as the prevention strategies was trained (Box 3). It was reported that iron overload impaired the immune system and increased the risk of infection in thalassemia patients (18). Moreover, an association was found between bacterial infection and iron and iron chelators. Many organisms, such as Yersinia enterocolitica, Klebsiella species, Escherichia coli, Streptococcus pneumoniae, 


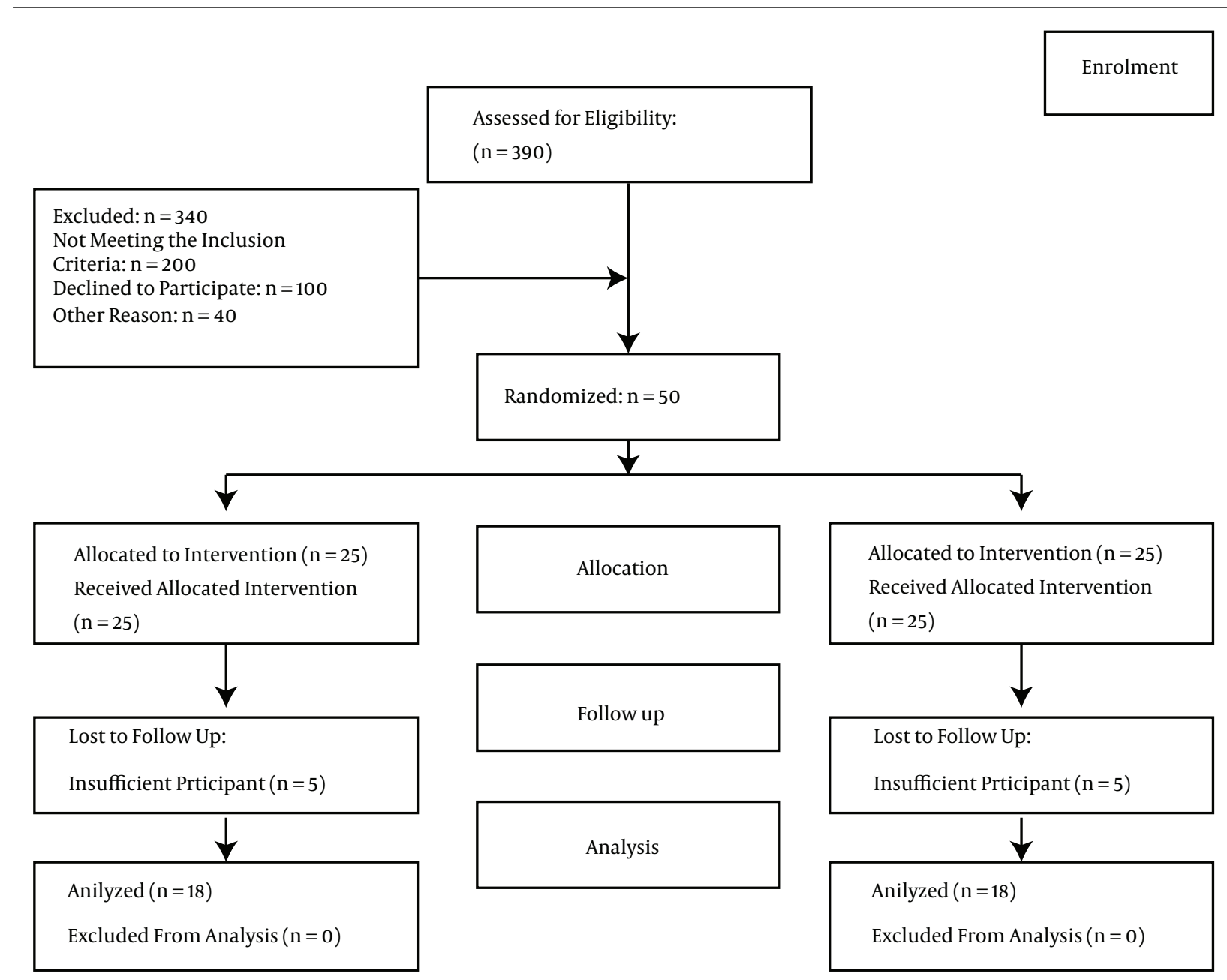

Figure 1. Consort Flow Diagram of the Participants of this Randomized Clinical Trial an Educational Program Designed to Reduce Iron Overload in Patients With betaThalassemia Major

Pseudomonas aeroginosa, Legionella pneumophila, and Listeria monocytogenes, have been indicated to increase virulence in the presence of excessive iron (19).

In the fifth session, the nature, reason, and ways of praying were explained and the patients were asked to pray every day and fill their moments with God by attracting their heart and mind towards Him. This program set out to focus on spirituality and creating tranquility in thalassemic patients. The spiritual training programs (including intercessory prayer) were carried out according to the participants' religious beliefs (all were Muslim). It should be mentioned that the patients were required to repeat spiritual words and phrases at least three times a day.

Each training class lasted for 1 - 1.5 hours considering the patients' understanding and tolerance levels. To ensure patient learning, they were involved in discussions and question-and-answer sessions after each training session. In addition, the participants were given a 15-page booklet entitled care program for major thalassemia patients, which contained the educational content of the training program after the intervention.

A self-reporting performance record form was filled out daily by the patients to confirm their compliance with the educational program. The patients completed the form every day for eight weeks. They recorded no intake of red meat, fish, liver and kidney, the consumption of dairy products but only with meals, having fruits and vegetables as snacks, walking and its duration, performing infection strategies, and frequency of repetition of spiritual phrases.

The control group received the routine trainings and cares. The researcher's contact number was also given to all the participants for any possible questions. 
Box 1. Dos and Don'ts of Eating in Thalassemia Patients

\section{Dos and Don'ts}

\section{Limitations in Meat Consumption}

Heme-iron-rich foods include meat, fish, and some parts of poultry and turkey (such as the breast and wings). The liver and kidneys are also rich sources of this type of iron. Therefore, it is better to limit the consumption of these foods. Moreover, using vegetable proteins and white instead of red meat is recommended.

Many foods contain non-heme-iron, including eggs, chocolate, cereals, vegetables, fruits, roots and tubers (like potatoes and carrots), dried fruits like raisins, peaches, plums, and figs. The absorption rate of these foods is much lower than that of heme-iron, and approximately $3-8$ percent of their iron is absorbed by the body.

The absorption of non-heme-iron is influenced by consuming other foods. Thus, serving some foods with non-heme-iron foods might increase or decrease the absorption of iron. Because non-heme-iron is present in many foods, it is difficult to avoid these foods. Red meat, poultry, fish, and other seafood contain large amounts of heme-iron and also increase the absorption of non-heme-iron from other foods.

Using Dairy

Milk, cheese, and yogurt reduce the absorption of iron. However, the calcium in these foods is necessary to prevent osteoporosis. Therefore, use milk, cheese, o yogurt in your diet. Compounds such as coffee and milk, milk and tea, cheesy sandwiches, cheesy spaghetti, yogurt, and rice and milk, are especially good for thalassemia patients.

\section{Bean Consumption}

Wheat, corn, oats, rice, and pulses such as soya, beans, lentils, and peas reduce the absorption of non-heme-iron. Thus, it is good to include a lot of cereals in the daily diet.

\section{Vitamin C}

Vitamin C is a factor that increases iron absorption. This vitamin is present in fruits (especially citrus fruits and tomatoes) and vegetables (parsley, watercress, fenugreek, leek, dill, cauliflower, bell peppers, etc.). Consuming an orange or $100 \mathrm{~g}$ of vegetables along with food increases the absorption of iron by two fold. Hence, it is better to avoid eating fruits and vegetables with or immediately after a meal. However, because fruits and vegetables contain a variety of vitamins and antioxidants and must be consumed, it is better to eat them between two meals (as a snack). Note: Although the consumption of vitamin C enhances iron absorption in these patients, some researchers have recommended a low intake of vitamin C along with desferal to aid in iron excretion.

Vitamin E

Iron overload can lead to cellular stress. Due to the effect of vitamin E on reducing cell damage, substances containing vitamin $\mathrm{E}$ are recommended. Foods rich in vitamin $\mathrm{E}$ include fruits, such as peaches and asparagus. Other sources of this vitamin also include plant materials, animal fat, vegetable oils, such as sunflower oil, olive, corn, peanuts, fresh nuts (hazelnuts, almonds, and walnuts), cabbage, soybean, and wheat germ oil.

Box 2. Exercise in Thalassemia Patients: Why and How?

\section{Exercise in Thalassemia Patients}

Doing some exercises based on one's tolerance reduces the bad effects of iron on the heart and improves the cardiovascular function. Regular exercise reduces iron overload in patients with thalassemia by decreasing their iron absorption from the gut.

Walk for at least 30 minutes on most days of the week and preferably all days of the week. Then, increase your time based on your tolerance. Also, enhance your speed gradually.

\section{Exercise Recommendations}

Gradually allow your body to get used to exercise

Start with easy exercises and then increase the intensity and duration gradually

Do gentle, soft, and warming exercises for 5 to 10 minutes. Gentle exercises reduce the possibility of heart diseases and sports injuries

Do not stop exercising at the end of the exercise suddenly. Decrease exercise gradually and then stop it

Avoid doing exercise in very cold, very hot, or humid weather. In warm weather, wear light and bright clothes. Avoid too tight clothes to allow heat loss

Use hats and sunscreen

Drink cold water before and after exercise

Be familiar with the signs of heart disease, such as chest, arm, hand, and jaw pain, nausea, dizziness, feeling faint, palpitations, and shortness of breath. In case you have these problems, stop exercising and go to the nearest health center

\subsection{Outcomes}

The outcomes were collected using the participants' demographic characteristics and basic iron content. The participants' demographic characteristics consisted of age, gender, education level, time of diagnosis, number of transfusions, and medications used to reduce iron over- 
Box 3. The Importance of Preventing Infection in Thalassemia Patients

Importance of Preventing Infection

There was an association between bacterial infection and iron and iron chelators

Prevention of Infection

Observe hand hygiene and wash your hands

Wash fruits and vegetables before use

Consult with your doctor about vaccines (pneumococcal vaccine, influenza, and hepatitis A and B)

Avoid swimming in rivers, lakes, and public pools

If you travel to other countries, consult with healthcare providers about the recommended immunization and health points (ask about the permission to drink water in glass bottles and avoid eating fruits, vegetables, and unpasteurized dairy)

Boil water fully in developing countries

Create a proper balance between activity and rest.

Report any symptoms of infection, including fever, chills, flushing, pain, redness, etc., to your doctor

Avoid contact with individuals with infectious diseases

load. Additionally, the basic iron content included serum iron, ferritin, unsaturated iron-binding capacity (UIBC), and total iron-inding capacity (TIBC).

The basic iron content was measured in a specialized laboratory. To ensure the reliability of the test results, all the experiments were carried out in a specialized laboratory by just one individual using specific kits (Electrochemi luminesence from Roche Germany) to determine the amount of ferritin and then the colorimetric method to determine the iron and UIBC at a given time. To prevent information transfer, the study was initially conducted in the control group, so that they could not get any information about the details of the intervention. The iron content was measured again after two months in the same specialized laboratory. It should be noted that the individual who took blood from the patients and the one who analyzed the tests were blind to the study groups.

\subsection{Ethical Considerations}

This study was registered in Iranian registry of clinical trials of the ministry of health and medical education (IRCT2014011216190N1). The study was also approved by the ethics committee of Shiraz University of Medical Sciences. Written informed consent was obtained from all the participants. In this way, they were informed about the study objectives and procedures. They were also informed about the voluntary nature of the study and that their participation/non-participation had no effects on their treatments and care. It was also emphasized that blood had to be taken from the participants. The patients were assured that their blood would be used only for study purposes.

\subsection{Data Analysis}

The study data were analyzed using chi-square, Fisher's exact test, Mann-Whitney, Wilcoxon, independent samples t-test, and paired samples t-test with the the SPSS statistical software (ver. 16). The significance level was set at 0.05 . The data were analyzed by a statistician who was blind to the study groups.

\section{Results}

Descriptive data of the two groups have been listed in Table 1 . The study results showed that the majority of the participants in both groups were 20 - 24 years old. In addition, $50 \%$ of the participants in the control group and $72.2 \%$ of those in the intervention group were female. In addition, $35 \%$ of the control group and $44.4 \%$ of the intervention group participants had high-school degrees. The results of the chi-square test (or Fisher's exact test in case the sample size was not large enough) showed that the subjects in the two groups were homogeneous in terms of demographic characteristics (age, sex, and education level), time of diagnosis, onset of transfusion, and chelator agent.

A comparison of the iron measurements in the two groups both before and after the intervention is presented in Table 2. As the tabulation depicts, the intervention group's mean of ferritin two months after the intervention was significantly lower compared to before the intervention $(\mathrm{P}=0.04)$. However, no significant change was observed in the control group in this regard $(\mathrm{P}=0.29)$. Besides, the mean level of serum iron was reduced in the intervention group, but the difference was not statistically 
Table 1. The Frequency Distribution of Demographic Variables, Time of Diagnosis, Chelator Agents, and Onset of Blood Transfusion in the Two Study Groups ${ }^{\mathrm{a}, \mathrm{b}}$

\begin{tabular}{|c|c|c|c|}
\hline Variable & Control Group, $(\mathbf{n}=\mathbf{2 0})$ & Intervention Group, $(\mathrm{n}=18)$ & P-Value ${ }^{c}$ \\
\hline Age Group & & & 0.75 \\
\hline $15-19$ & $3(15)$ & $1(5.6)$ & \\
\hline $20-24$ & $7(35)$ & $9(50)$ & \\
\hline $25-29$ & $7(35)$ & $6(33.3)$ & \\
\hline $30-35$ & $3(15)$ & $2(11.1)$ & \\
\hline Gender & & & 0.16 \\
\hline Female & $10(50)$ & $13(72.2)$ & \\
\hline Male & $10(50)$ & $5(27.8)$ & \\
\hline Education Level & & & 0.35 \\
\hline Illiterate & $0(0)$ & $0(0)$ & \\
\hline Elementary school & $0(0)$ & $3(16.7)$ & \\
\hline Middle school & $5(25)$ & $3(16.7)$ & \\
\hline High school & $3(15)$ & $2(11.1)$ & \\
\hline Diploma & $7(35)$ & $8(44.4)$ & \\
\hline Academic & $5(25)$ & $2(11.1)$ & \\
\hline Time of Diagnosis, month & & & 0.95 \\
\hline $0-6$ & $11(55)$ & $9(50)$ & \\
\hline $7-12$ & $4(20)$ & $5(27.8)$ & \\
\hline $13-18$ & $1(5)$ & $0(0)$ & \\
\hline $19-24$ & $4(20)$ & $4(22.2)$ & \\
\hline Onset of Blood Transfusion, months & & & 0.56 \\
\hline $0-6$ & $10(50)$ & $6(33.3)$ & \\
\hline $7-12$ & $5(25)$ & $7(38.9)$ & \\
\hline $13-18$ & $1(5)$ & $0(0)$ & \\
\hline $19-24$ & $4(20)$ & $5(27.8)$ & \\
\hline Chelator Agent & & & 0.18 \\
\hline Desferal & $5(25)$ & $3(16.7)$ & \\
\hline Osveral & $4(20)$ & $1(5.6)$ & \\
\hline Desfonak & $0(0)$ & $1(5.6)$ & \\
\hline Deferiprone & $2(10)$ & $6(33.3)$ & \\
\hline Exjade & $3(15)$ & $0(0)$ & \\
\hline Desferal + Osveral & $0(0)$ & $1(5.6)$ & \\
\hline Desferal + Deferiprone & $5(25)$ & $3(16.7)$ & \\
\hline Osveral + Desfonak & $0(0)$ & $1(5.6)$ & \\
\hline Desfonak + Deferiprone & $1(5)$ & $1(5.6)$ & \\
\hline Desferal + Exjade & $0(0)$ & $1(5.6)$ & \\
\hline
\end{tabular}

significant $(\mathrm{P}=0.81)$. Also, this index increased in the control group, but the difference was not statistically significant $(P=0.4)$. Moreover, the UIBC level decreased in both groups, but it the decrease was significant only in the control group $(\mathrm{P}=0.04)$ compared to the intervention group $(\mathrm{P}=0.32)$. The TIBC level also decreased in both the intervention and control groups, but the difference was not statistically significant ( $\mathrm{P}=0.26$ and $\mathrm{P}=0.11$, respectively). Also, despite the decline in ferritin level in the intervention group after the intervention, the difference was not statis- tically significant $(\mathrm{P}=0.96)$; however, it was clinically considerable. Finally, changes in serum iron, UIBC, and TIBC levels were not statistically significant $(\mathrm{P}=0.96, \mathrm{P}=0.12$, and $\mathrm{P}=0.20$, respectively). A comparison of the changes in iron indices in the two groups before and after the intervention has been presented in Table 3.

\section{Discussion}

The results of this study showed that application of a comprehensive educational program could improve fer- 
Table 2. A Comparison of the Mean Iron Indices in the Study Groups Both Before and After the Intervention ${ }^{\mathrm{a}, \mathrm{b}}$

\begin{tabular}{|c|c|c|c|c|c|c|}
\hline \multirow[t]{2}{*}{ Variables } & \multicolumn{2}{|c|}{ Control Group } & \multirow[t]{2}{*}{ P-Value ${ }^{c}$} & \multicolumn{2}{|c|}{ Intervention Group } & \multirow[t]{2}{*}{ P-Value } \\
\hline & Before the Intervention & $\begin{array}{l}\text { Two months After the In- } \\
\text { tervention }\end{array}$ & & Before the Intervention & $\begin{array}{l}\text { Two Months After the In- } \\
\text { tervention }\end{array}$ & \\
\hline Serum iron, $\mu \mathbf{g} / \mathbf{d L}$ & $162.84(27.53)$ & $167.94(36.68)$ & 0.4 & $168.5(22.99)$ & $167.37(31.57)$ & 0.81 \\
\hline Ferritin, ng/mL & $1721.88(1132.04)$ & $1815.82(1149.9)$ & 0.29 & $2275.18(2369.1)$ & $1936.65(1936.4)$ & 0.04 \\
\hline UIBC, mg/dL & $41.86(82.59)$ & $16.47(42.23)$ & $0.04^{\mathrm{d}}$ & $80.81(131.80)$ & $46.66(108.83)$ & $0.32^{\mathrm{d}}$ \\
\hline TIBC, $\mu \mathrm{g} / \mathbf{d L}$ & $207.38(93.48)$ & $184.42(61.48)$ & 0.26 & $225.09(144.58)$ & $222.55(112.88)$ & 0.11 \\
\hline
\end{tabular}

Abbreviations: SD, standard deviation; TIBC, total iron binding capacity; UIBC, unsaturated iron binding capacity.

${ }^{\mathrm{a}}$ Values are expressed as mean (SD).

bignificance level: 0.05 .

cpaired-samples t-test.

${ }^{\mathrm{d}}$ Mann-Whitney test.

Table 3. A Comparison of the Changes in the Iron Indices in the Study Groups Both Before and After the Intervention

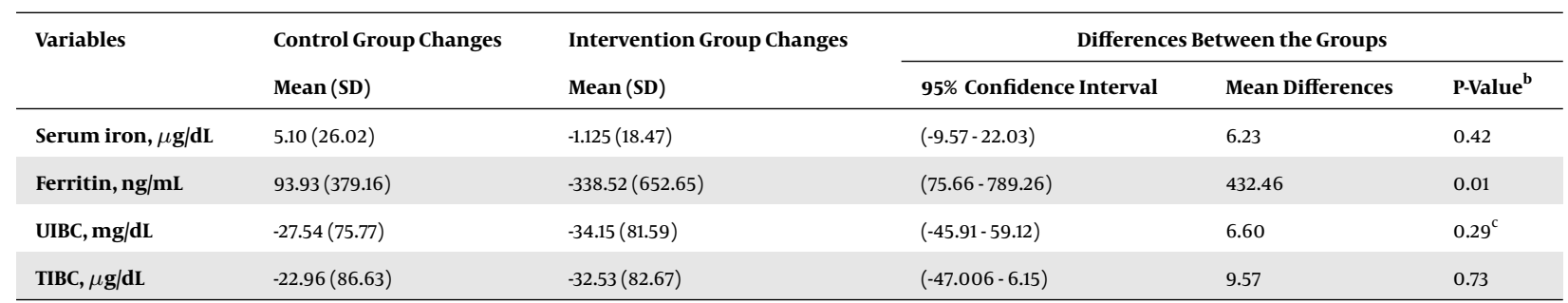

Abbreviations: UIBC, unsaturated iron binding capacity; TIBC, total iron binding capacity; SD, standard deviation.

a Significance level: 0.05 .

${ }^{\mathrm{b}}$ Independent-samples t-test.

${ }^{\mathrm{c}}$ Mann-Whitney test.

ritin levels and eventually decrease the complications of iron overload in thalassemic patients. In fact, application of the educational program in the intervention group significantly decreased the serum ferritin level two months following the intervention in comparison to before the intervention. This observation was consistent with the findings of the study by Rahmaninia, as cited in Heidary, which was conducted on the athletes in order to compare the effects of two types of physical activities on iron status and the soluble transferrin receptor (2). Presumably, the insignificant reduction in the intervention group participants' serum iron levels was due to daily changes in serum iron levels as well as to demolition of iron release from red blood cells. In general, serum iron fluctuates daily and is influenced by several factors, including diet and infectious or inflammatory diseases $(15,20)$. These results were also in line with those obtained by Heidary et al. in a study investigating the effect of eight weeks of aerobic training on hematology indices in patients with beta-thalassemia minor. The study results indicated that the serum ferritin level significantly was reduced in the intervention group eight weeks after doing aerobic exercises (2).
The present study further attempted to investigate the effects of exercising in conjunction with other methods of iron overload reduction, such as the reduction of intestinal iron absorption through the consumption or prohibition of some food groups, the application of safety methods to prevent infectious diseases, and prayer. Considering the effect of exercising on reducing ferritin, several mechanisms have been proposed. It seems reasonable that hemolysis of unfavorable thalassemic hemoglobin occurs more frequently during exercise activities, eventually decreasing serum ferritin levels. Additionally, some researchers believe that intestinal iron absorption is lower than normal in athletes. Hence, in case thalassemic patients can decrease iron absorption through regular and constant aerobic activities, they can control their iron supply (16).

Exercising results in an increase in the percentage of iron autolysis from tissues. Changes in the cell membrane, in turn, release tissue iron and increase serum ferritin and iron levels. However, desferal helps the iron to be excreted when iron is present in the blood. This is where the role of exercise in reduction of iron overload is manifested either directly or indirectly by increasing the efficiency of 
desferal (21).

The findings of the present study showed a decreased UIBC in both study groups, but the difference was significant only in the control group $(\mathrm{P}<0.05)$. Studies have shown that high degrees of some substances, such as bilirubin, copper, and lipemia, and hemoglobin hemolysis $>200 \mathrm{mg} / \mathrm{dL}$ could affect UIBC; hence, the obtained results might not be very reliable (22). On the other hand, both UIBC and TIBC measure the capacity of transferrin. Transferrin is a protein that is produced by the liver. Thus, in case of liver disease or lack of adequate dietary protein, transferrin levels are reduced (23) and, consequently, both UIBC and TIBC drop without any direct relationship to the iron changes.

Although serum iron dropped in the present study, TIBC did not increase. These findings were not consistent with those of the studies by Heydari et al. and Vashtani et al. $(2,16)$. In addition to what was mentioned earlier about UIBC, increase of TIBC following reduction of serum iron level mainly occurs in anemia. In iron overload, on the other hand, by saturation of transferrin-iron binding capacity, non-transferrin bound iron (NTBI), which is more toxic compared to iron bound to transferrin, appears in the blood circulation $(4,24,25)$. Therefore, the change in TIBC in response to a reduction of available serum iron is not valid in patients with iron overload.

One of the limitations of the current study was that the patients' responsibility and economic, occupational, and social status could have affected their adherence to and compliance with the training. Therefore, to achieve more accurate results, further studies with larger sample sizes, longer follow-up periods, and more accurate methods of assessing iron overload (such as soluble transferrin receptor liver iron concentration) are recommended to be carried out.

Nowadays, similar to other patients suffering from other chronic diseases, thalassemic patients require careful training to perform the required care. Providing and regulating training and care programs through comprehensive and constant courses by nurses can result in more accurate follow-ups and help reduce iron overload. These programs should be carried out by nurses since they have direct contact with patients while providing their services. In addition, comprehensive care programs meet the patients' educational needs in various dimensions; evidence from experiments has expressed the efficiency of such programs in reducing ferritin in thalassemic patients. Therefore, the application of such care programs is recommended as a crucial step in reducing iron overload and its complications in different bodily organs, thereby improving the thalassemic patients' health status.

\section{Acknowledgments}

The authors would like to thank Ms. A. Keivanshekouh at the research improvement center of Shiraz University of Medical Sciences for improving the use of English in the manuscript.

\section{Footnote}

Funding/Support: This study was extracted from the M.Sc. thesis written by Roghaye Noormohammadi and funded by Shiraz University of Medical Sciences (Grant No. 905782).

\section{References}

1. Muncie HJ, Campbell J. Alpha and beta thalassemia. Am Fam Physician 2009;80(4):339-44. [PubMed: 19678601].

2. Heidary H, Bijeh N, Javahery SAAH, Abrishami F. The effect of eight weeks of aerobic training on hematological indices in $\beta$-thalassemia minor patients in kermanshah. Horiz Med Sci. 2011;17(3):20-8.

3. Galanello R, Origa R. Beta-thalassemia. Orphanet J Rare Dis. 2010;5:11. doi: 10.1186/1750-1172-5-11. [PubMed: 20492708].

4. Kohgo Y, Ikuta K, Ohtake T, Torimoto Y, Kato J. Body iron metabolism and pathophysiology of iron overload. Int J Hematol. 2008;88(1):7-15. doi: 10.1007/s12185-008-0120-5. [PubMed: 18594779].

5. Patel HV, Qari M, Mousa SA, Bloemen S, Laat BD, Hemker HC, et al. Iron balance in $\beta$-thalassemia: Maintaining an antioxidant/oxidant ratio. JAppl Hematol. 2012;3(1):4.

6. Gujja P, Rosing DR, Tripodi DJ, Shizukuda Y. Iron overload cardiomyopathy: better understanding of an increasing disorder. $J$ Am Coll Cardiol. 2010;56(13):1001-12. doi: 10.1016/j.jacc.2010.03.083. [PubMed: 20846597].

7. Waseem F, Khemomal KA, Sajid R. Antioxidant status in beta thalassemia major: a single-center study. Indian J Pathol Microbiol 2011;54(4):761-3. doi: 10.4103/0377-4929.91489. [PubMed: 22234105].

8. Claster S, Wood JC, Noetzli L, Carson SM, Hofstra TC, Khanna $\mathrm{R}$, et al. Nutritional deficiencies in iron overloaded patients with hemoglobinopathies. Am J Hematol. 2009;84(6):344-8. doi: 10.1002/ajh.21416. [PubMed: 19415722].

9. Fung EB. Nutritional deficiencies in patients with thalassemia. Ann N Y Acad Sci. 2010;1202:188-96. doi: 10.1111/j.1749-6632.2010.05578.x. [PubMed: 20712792].

10. Lee JW. Iron chelation therapy in the myelodysplastic syndromes and aplastic anemia: a review of experience in South Korea. Int J Hematol. 2008;88(1):16-23. doi: 10.1007/s12185-008-0117-0. [PubMed:18604581].

11. Marsella M, Pepe A, Borgna-Pignatti C. Better survival and less cardiac morbidity in female patients with thalassemia major: a review of the literature. Ann NYAcad Sci. 2010;1202:129-33. doi: 10.1111/j.17496632.2010.05588.x. [PubMed: 20712783].

12. Wang Y, Wu M, Al-Rousan R, Liu H, Fannin J, Paturi S, et al. Iron-induced cardiac damage: role of apoptosis and deferasirox intervention. J Pharmacol Exp Ther. 2011;336(1):56-63. doi: 10.1124/jpet.110.172668. [PubMed: 20947636].

13. Britton RS, Leicester KL, Bacon BR. Iron toxicity and chelation therapy. Int J Hematol. 2002;76(3):219-28. [PubMed: 12416732].

14. Yang LP, Keam SJ, Keating GM. Deferasirox : a review of its use in the management of transfusional chronic iron overload. Drugs. 2007;67(15):2211-30. [PubMed: 17927285]. 
15. Schumacher YO, Schmid A, Konig D, Berg A. Effects of exercise on soluble transferrin receptor and other variables of the iron status. $B r J$ Sports Med. 2002;36(3):195-9. [PubMed:12055114].

16. Vashtani SH, Nazem F, Bordar F. The effect of aerobic rehabilitation program on concentration of ferritin, iron, TIBC and cardiovascular operation in the young patients suffering from major thalassemia. $J$ Guilan Univ Med Sci. 2009;18(71):95-102.

17. Rashidi M, Aboomardani M, Rafraf M, Arefhosseini SR, Keshtkar A, Joshaghani H. Effects of Vitamin E and Zinc Supplementation on Antioxidants in Beta thalassemia major Patients. Iran J Pediatr. 2011;21(1):8-14. [PubMed: 23056757].

18. Mishra AK, Tiwari A. Iron overload in Beta thalassaemia major and intermedia patients. Maedica (Buchar). 2013;8(4):328-32. [PubMed: 24790662].

19. Cappellini MD, Cohen A, Eleftheriou A, Piga A, Porter J, Taher A. In: Guidelines for the Clinical Management of Thalassaemia. 2nd revised ed. Cappellini MD, Cohen A, Eleftheriou A, Piga A, Porter J, Taher A, editors. Nicosia (CY): Thalassaemia International Federation; 2008. Infections in Thalassaemia Major.

20. Hawkins RC. Total iron binding capacity or transferrin concentration alone outperforms iron and saturation indices in pre- dicting iron deficiency. Clin Chim Acta. 2007;380(1-2):203-7. doi: 10.1016/j.cca.2007.02.032. [PubMed: 17376421].

21. Jordan J, Kiernan W, Merker HJ, Wenzel M, Beneke R. Red cell membrane skeletal changes in marathon runners. Int J Sports Med. 1998;19(1):16-9. doi:10.1055/s-2007-971873. [PubMed: 9506794].

22. Ji JZ, Meng QH. Evaluation of the interference of hemoglobin, bilirubin, and lipids on Roche Cobas 6000 assays. Clin Chim Acta. 2011;412(17-18):1550-3. doi: 10.1016/j.cca.2011.04.034. [PubMed: 21575617].

23. Wish JB. Assessing iron status: beyond serum ferritin and transferrin saturation. Clin J Am Soc Nephrol. 2006;1 Suppl 1:S4-8. doi: 10.2215/CJN.01490506. [PubMed: 17699374].

24. Ito S, Ikuta $K$, Kato $D$, Shibusa $K$, Niizeki $N$, Tanaka $H$, et al. Non-transferrin-bound iron assay system utilizing a conventional automated analyzer. Clin Chim Acta. 2014;437:129-35. doi: 10.1016/j.cca.2014.07.013. [PubMed: 25072389].

25. Brissot P, Ropert M, Le Lan C, Loreal O. Non-transferrin bound iron: a key role in iron overload and iron toxicity. Biochim Biophys Acta. 2012;1820(3):403-10. doi: 10.1016/j.bbagen.2011.07.014. [PubMed: 21855608]. 